\title{
Importance of Adjunctive Heart Failure Optimization Immediately After Implantation to Improve Long-Term Outcomes With Cardiac Resynchronization Therapy
}

\author{
Wilfried Mullens, MD, PhD ${ }^{\mathrm{a}, \mathrm{b}, *}$, Jacek Kepa, MS ${ }^{\mathrm{b}}$, Philippe De Vusser, MD ${ }^{\mathrm{a}}$, Jan Vercammen, $\mathrm{RN}^{\mathrm{a}}$, \\ Maximo Rivero-Ayerza, MD, PhD ${ }^{\mathrm{a}}$, Patrick Wagner, $\mathrm{PhD}^{\mathrm{b}}$, Joseph Dens, MD, $\mathrm{PhD}^{\mathrm{a}}$, \\ Mathias Vrolix, MD ${ }^{\mathrm{a}}$, Pieter Vandervoort, MD ${ }^{\mathrm{a}}$, and W.H. Wilson Tang, $\mathrm{MD}^{\mathrm{c}}$
}

Despite improvement in morbidity and mortality with cardiac resynchronization therapy (CRT), disease progression continues to affect a subset of patients and there is limited effort to identify contributing factors. Our objective was to investigate if a protocol-driven approach incorporated in a management strategy of heart failure immediately after implantation would provide incremental benefits beyond usual care after implantation. We reviewed 114 consecutive patients with CRT implanted from 2005 through 2009 who received usual care after implantation or underwent protocol-driven CRT care after implantation. Preimplantation characteristics in patients receiving usual versus protocoldriven care were similar in left ventricular (LV) dimension ( $\mathrm{LV}$ internal diastolic diameter $6.2 \pm 0.8$ vs $6.4 \pm 1.0 \mathrm{~cm})$, LV ejection fraction $(26 \pm 8 \%$ vs $25 \% \pm 8 \%)$, QRS width, and medication usage. Major adjustments during the protocol-driven approach were uptitration of neurohormonal blockers (64\%), echocardiographically guided atrioventricular optimization (50\%), heart failure education (42\%), arrhythmia management (19\%), and LV lead repositioning (7\%). Although positive LV remodeling was noted in the 2 groups at 6 months, extent was significantly greater in the protocol-driven approach compared to usual care (change in LV internal diastolic diameter $0.7 \pm 0.6 \mathrm{~cm}$ vs $0.2 \pm 1.2 \mathrm{~cm}, \mathrm{p}=0.01$; change in LV ejection fraction $11 \pm 7 \%$ vs $7 \pm 9 \%, p=0.01$ ), which was associated with fewer major adverse events (14\% vs $53 \%$, p <0.001). In conclusion, a protocol-driven approach for patients with CRT started immediately after implantation is associated with incremental favorable effects on reverse remodeling and fewer adverse events compared to usual care after implantation. These effects appeared to be driven not only by changes in device settings and arrhythmia management but also by concomitant medication optimization and heart failure education. (C) 2011 Elsevier Inc. All rights reserved. (Am J Cardiol 2011;108:409-415)

Most therapeutic effects of cardiac resynchronization therapy (CRT) have been attributed to device-induced decrease in dyssynchrony. For those who do not demonstrate noticeable improvements immediately after implantation, further optimization of specific device programming, arrhythmia management, and considerations for lead repositioning, if suboptimal, can be beneficial. ${ }^{1}$ However, it is often assumed that only those who did not respond over time would benefit from such optimization strategies because the maximal therapeutic effect of CRT should be derived if implantation and device programming are optimal. Factors favorably or adversely affecting CRT efficacy, particularly those in the immediate postimplantation setting, have not been extensively studied. Therefore, the objective

\footnotetext{
${ }^{a}$ Department of Cardiovascular Medicine, Ziekenhuis Oost Limburg, Genk, Belgium; 'bchool of Life Sciences, Transnational University Limburg, Belgium; ' ${ }^{\circ}$ Department of Cardiovascular Medicine, Heart and Vascular Institute, Cleveland Clinic, Cleveland, Ohio. Manuscript received January 12, 2011; revised manuscript received and accepted March 22, 2011.

*Corresponding author: Tel: 32-8932-7087; fax: 32-8932-7918.

E-mail address: wilfried.mullens@zol.be (W. Mullens).
}

of this study was to investigate if a protocol-driven approach incorporated in a management strategy of heart failure immediately after implantation would provide incremental benefits beyond usual care after implantation including changes in exercise and echocardiographic parameters and long-term adverse cardiac events.

\section{Methods}

This study included consecutive patients with CRT using an implanted pacemaker or defibrillator under standard clinical indications in a single tertiary cardiac care institution from November 2005 through February 2010. All patients demonstrated stable but advanced heart failure symptoms (New York Heart Association functional class III or IV symptoms) despite receiving optimal medical therapy as tolerated by the patient, decreased left ventricular (LV) ejection fraction $(\leq 35 \%)$, and prolonged QRS duration $(\geq 120 \mathrm{~ms})$ at time of implantation. Because of stringent reimbursement criteria in Belgium, CRT using implanted defibrillators was performed only cases of previous episodes of sustained ventricular tachycardia or inducible ventricular 


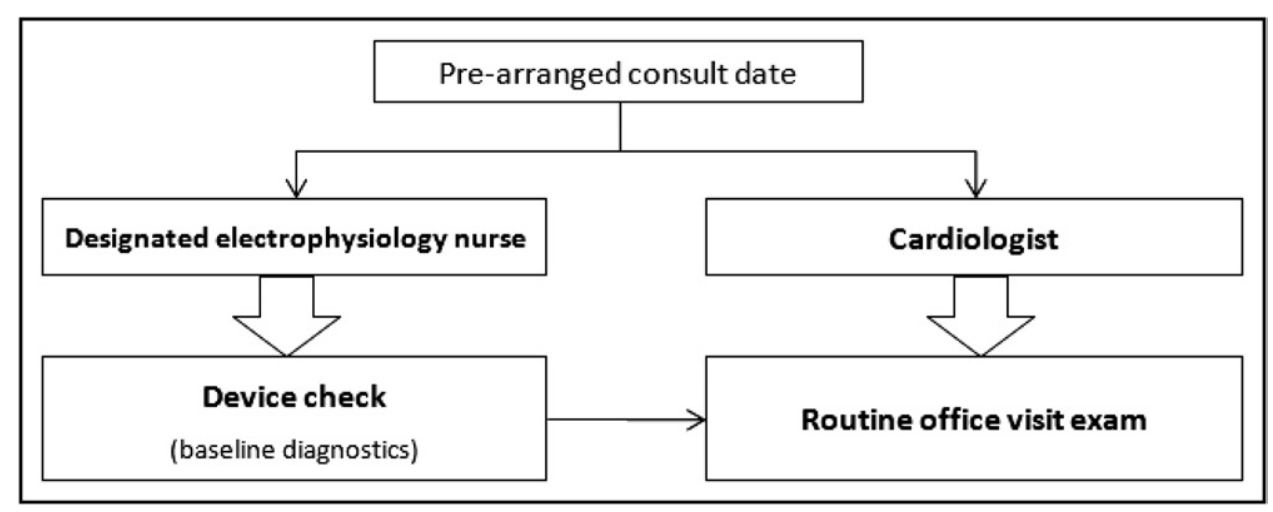

Figure 1. Flow chart presenting usual care after implantation.

arrhythmia. The study complied with the Declaration of Helsinki, the locally appointed ethics committee approved the research protocol, and informed consent was obtained from the subjects.

A protocol-driven CRT optimization protocol was established in August 2008; thus patients were stratified in 2 groups according to CRT implantation before August 2008 ("usual care") versus after August 2008 ("CRT optimization protocol"). Before August 2008 patients received usual care after implantation performed generally by different hospital staffs. In this model, an electrophysiology nurse performed a standard device check. Afterward a patient was seen by a treating cardiologist to assess the patient's current health status and symptoms, often also performing echocardiography (Figure 1). Changes in device settings and heart failure therapy were at the discretion of the treating cardiologist.

A postimplantation stepwise CRT optimization protocol was established as part of a multidisciplinary approach toward postimplantation CRT care incorporated in management program of heart failure in August 2008. In the CRT optimization protocol, number of scheduled clinic visits was similar to that of the usual-care group (6 weeks, 3 and 6 months after implantation). Beyond the standard device check, a more thorough CRT optimization clinic protocol was conducted, which included a wider variety of measurements and prespecified optimization guidelines performed in a designated clinic staffed with physicians and nurses with a broad interest in heart failure and cardiac devices as previously described (Figure 2). ${ }^{1}$ In summary, a heart failure nurse recorded an electrocardiogram to assess heart rate, QRS width, and AV/PR intervals. This was performed 2 times with the implanted pacemaker turned on and off to ensure adequate biventricular pacing. Next, an anteriorposterior and lateral chest $\mathrm{x}$-ray was carried out to determine optimal positioning of the right atrial, right ventricular, and LV leads (in basal or midlateral and posterior position). In the meantime, routine laboratory tests were done to detect occult hematologic and metabolic derangements. After these measurements, the designated cardiologist recorded a detailed history on heart failure symptoms, occurrence of arrhythmias, and potential device-related issues, checked for compliance to medication usage and salt/ fluid restriction, and completed a full physical cardiovascular examination.
Afterward a comprehensive 2-dimensional echocardiographic examination was performed (Philips Medical Systems, Andover, Massachusetts) with nominal settings of the CRT device. All reported echocardiographic measurements including LV size/function and mitral regurgitation were averaged from $\geq 3$ consecutive cycles as recommended by the American Society of Echocardiography. ${ }^{2}$ Then an effort was made to optimize LV diastolic filling when it differed from stage I by altering AV timing using conventional Doppler echocardiography. Optimal AV interval was determined by sampling mitral inflow with pulse-wave Doppler to correspond with the shortest $\mathrm{AV}$ interval that dissociated the $\mathrm{E}$ and $\mathrm{A}$ waves but did not interrupt the end of the $\mathrm{A}$ wave. $^{3-5}$

To evaluate a patient's physical fitness and ensure biventricular pacing was persistent even during exercise, a cycloergometric bicycle test with maximum oxygen uptake recording was performed. Based on the findings, a recommendation was proposed to the patient to maximize the potential of CRT. These recommendations were not mutually exclusive because actions could be categorized by repositioning of the LV lead to correct inappropriate lead positioning, changes in device programming for suboptimal device programming (mostly AV timing), or treatment of arrhythmias medically or invasively.

Thorough efforts were taken toward optimization of medical therapy, i.e., uptitration of neurohormonal blockers to guideline-recommended doses, which were often not tolerated before implantation. In addition, adequate heart failure education was provided to familiarize a patient with heart failure risk factors and modifications in lifestyle. Patients were informed through dietary consults about salt-free diets (2 to $3 \mathrm{~g} /$ day) and fluid restriction (1 to $1.5 \mathrm{~L} /$ day), which often coincided with a progressive decrease in loop diuretic doses. Importantly, all these adjustments were implemented in close collaboration with general practitioners who were informed through telephone contact the day of the patient's clinic visit and provided with the findings and recommendations of the clinic through an on-line letter sent immediately after the CRT clinic visit to ensure optimization of medical therapy was accomplished at home under close supervision.

We prespecified the primary end points for analysis as time to first occurrence of any of the following: all-cause mortality, cardiac transplantation, and/or first readmission 


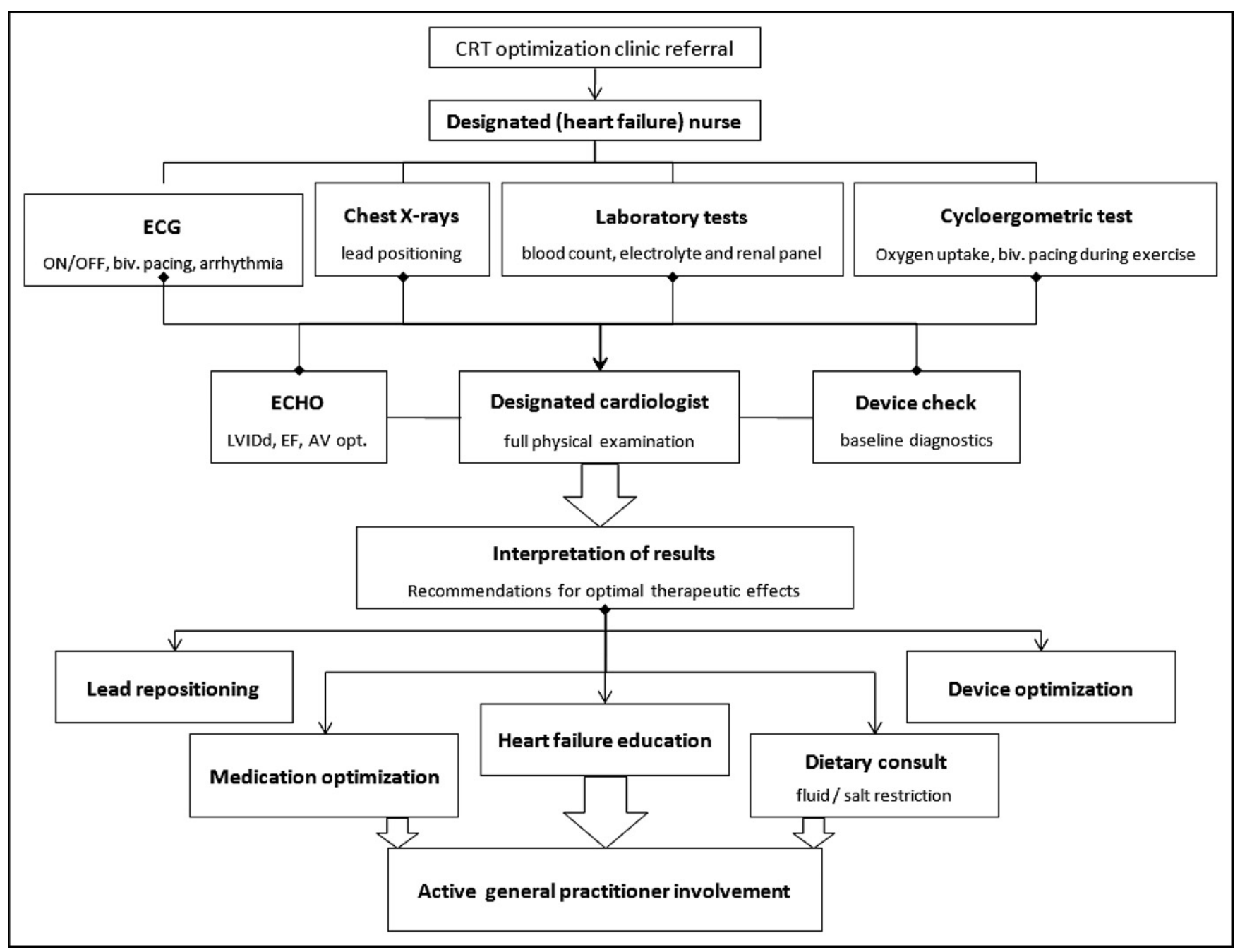

Figure 2. Flow chart presenting protocol-driven postimplantation management in cardiac resynchronization therapy clinic. AV opt. $=$ atrioventricular optimization; biv. = biventricular; ECG = electrocardiography; ECHO = echocardiography; $\mathrm{EF}=$ ejection fraction; LVIDd = left ventricular internal diastolic diameter.

for heart failure after implantation. Patients in the usual-care group were followed from implantation to the date of the first visit to the protocol-driven care clinic, whereas patients in the protocol-driven group were followed until April 30, 2010.

Collected data are expressed as mean \pm SD for continuous data and as ratio for categorical data. Paired-sample $t$ tests were performed for variables between related patient data groups and independent-sample $t$ tests were performed for variables between unrelated patient data groups. Statistical significance was set at a 2-tailed probability level with alpha equal to 0.05 . Kaplan-Meier survival curves were calculated with combined end points for all patients stratified in 2 groups. Cox proportional hazards regression model was used to determine which variables were related significantly to the different end point during the follow-up period. The authors had full access to the data and take responsibility for the integrity of the data. All authors have read and agreed to the report as written. All statistical analyses were performed using SPSS 17.0 for Windows (SPSS, Inc., Chicago, Illinois).

\section{Results}

Preimplantation patient characteristics are presented in Table 1 and were similar in the 2 groups $(n=53$ in usual-care group and 61 in protocol-driven group) including degree of LV remodeling and maximum exercise capacity. There was a large and similar proportion of use of neurohormonal blockers. For the CRT optimization group, mean clinic visit duration was 40 minutes with involvement of a designated nurse $( \pm 20$ minutes) and a cardiologist $( \pm 20$ minutes).

All patients had a lead implanted in the right atrium, right ventricle, and left ventricle through the coronary sinus $(72 \%)$ or epicardially (28\%). X-rays demonstrated no lead dislodgement but indicated a suboptimal positioning of the $\mathrm{LV}$ lead for 8 patients. One patient was scheduled for LV lead repositioning.

Device interrogation was successful in all patients, which was paced mostly in an atrial sensing-ventricular pacing mode. No battery depletion or lead integrity was noted. 
Table 1

Baseline demographics

\begin{tabular}{|c|c|c|c|c|}
\hline & $\begin{array}{c}\text { Total } \\
(\mathrm{n}=114)\end{array}$ & $\begin{array}{l}\text { Usual Care } \\
(\mathrm{n}=51)\end{array}$ & $\begin{array}{l}\text { Protocol-Driven } \\
\qquad(\mathrm{n}=63)\end{array}$ & $\mathrm{p}$ Value \\
\hline \multicolumn{5}{|l|}{ Demographics } \\
\hline Men & $64 \%$ & $55 \%$ & $73 \%$ & 0.04 \\
\hline Cardiac resynchronization therapy with defibrillator & $51 \%$ & $52 \%$ & $48 \%$ & NS \\
\hline Weight (kg) & $77 \pm 17$ & $76 \pm 16$ & $79 \pm 17$ & NS \\
\hline Body mass index $\left(\mathrm{kg} / \mathrm{m}^{2}\right)$ & $28 \pm 5$ & $27 \pm 5$ & $28 \pm 5$ & NS \\
\hline Hypertension $(>140 / 90 \mathrm{~mm} \mathrm{Hg})$ & $42 \%$ & $40 \%$ & $43 \%$ & NS \\
\hline Hyperlipidemia (low-density lipoprotein $>110 \mathrm{mg} / \mathrm{dl}$ ) & $33 \%$ & $28 \%$ & $37 \%$ & NS \\
\hline Quit smoking & $20 \%$ & $21 \%$ & $18 \%$ & NS \\
\hline Active smoking & $10 \%$ & $12 \%$ & $9 \%$ & NS \\
\hline Diabetes mellitus & $30 \%$ & $30 \%$ & $30 \%$ & NS \\
\hline$\beta$ Blockers & $89 \%$ & $82 \%$ & $94 \%$ & NS \\
\hline Spironolactone & $58 \%$ & $39 \%$ & $73 \%$ & 0.001 \\
\hline Loop diuretic & $75 \%$ & $84 \%$ & $67 \%$ & 0.02 \\
\hline Statin & $59 \%$ & $55 \%$ & $62 \%$ & NS \\
\hline Hydralazine & $9 \%$ & $2 \%$ & $14 \%$ & 0.02 \\
\hline Isosorbide dinitrate & $6 \%$ & $2 \%$ & $10 \%$ & NS \\
\hline Digoxin & $24 \%$ & $24 \%$ & $24 \%$ & NS \\
\hline \multicolumn{5}{|l|}{ Electrocardiographic data } \\
\hline Heart rate (beat/min) & $72 \pm 21$ & $75 \pm 19$ & $70 \pm 22$ & NS \\
\hline PR width (ms) & $192 \pm 47$ & $192 \pm 48$ & $191 \pm 44$ & NS \\
\hline QRS width (ms) & $159 \pm 31$ & $155 \pm 31$ & $160 \pm 31$ & NS \\
\hline \multicolumn{5}{|l|}{ Cycloergometric data } \\
\hline Maximum exercise capacity (watts) & $89 \pm 32$ & $84 \pm 22$ & $90 \pm 34$ & NS \\
\hline Tricuspid valve regurgitation (grade $>$ II) & $3 \%$ & $2 \%$ & $3 \%$ & NS \\
\hline
\end{tabular}

Arrhythmias were present in $19 \%$ of patients, mostly atrial fibrillation, but in 7\% frequent ventricular ectopy was present, leading to $<100 \%$ biventricular pacing in $19 \%$ and $<90 \%$ in $17 \%$ of patients. All arrhythmias were treated accordingly at least to ensure $>90 \%$ biventricular pacing in $95 \%$ instead of $83 \%$ of patients.

An additional $50 \%$ of patients were found to be programmed with suboptimal AV timing settings. AV timings were always optimized in these patients after an improvement in LV filling. These improvements were confirmed during the next clinic visit, with only 2 patients needing an additional change in their AV timings.

Uptitration in neurohormonal blockers was possible for $64 \%$ of patients, although $>90 \%$ were already receiving angiotensin-converting enzyme inhibitor and $\beta$-blocker medication at time of implantation. Interestingly, all these patients were already taking a similar dosage of neurohormonal blockers for $>3$ months before device implantation and only noted an improved tolerance toward uptitration of these drugs after implantation (Figure 3).

Failure in patient compliance with regard to salt/water restriction and stringent intake of medication was high because $42 \%$ patients testified to having poorly followed their daily medication intake and dietary advice. After implementation of heart failure education and dietary consult, in collaboration with general practitioners $22 \%$ of patients had a decrease in dosage of loop diuretic (Figure 4).

In addition, $63 \%$ patients were obese, $13 \%$ of patients had anemia, but only 1 patient had hemoglobin $<10 \mathrm{~g} / \mathrm{dl}$, which was treated with transfusion or erythropoietin agents.

Starting from August 2008 all patients receiving usual care were also referred to the protocol-driven CRT clinic. Interestingly, clinical, electrophysiologic, and device-related interventions were similar overall compared to patients followed in the protocol-driven clinic immediately after implantation. Indeed, uptitration of neurohormonal 


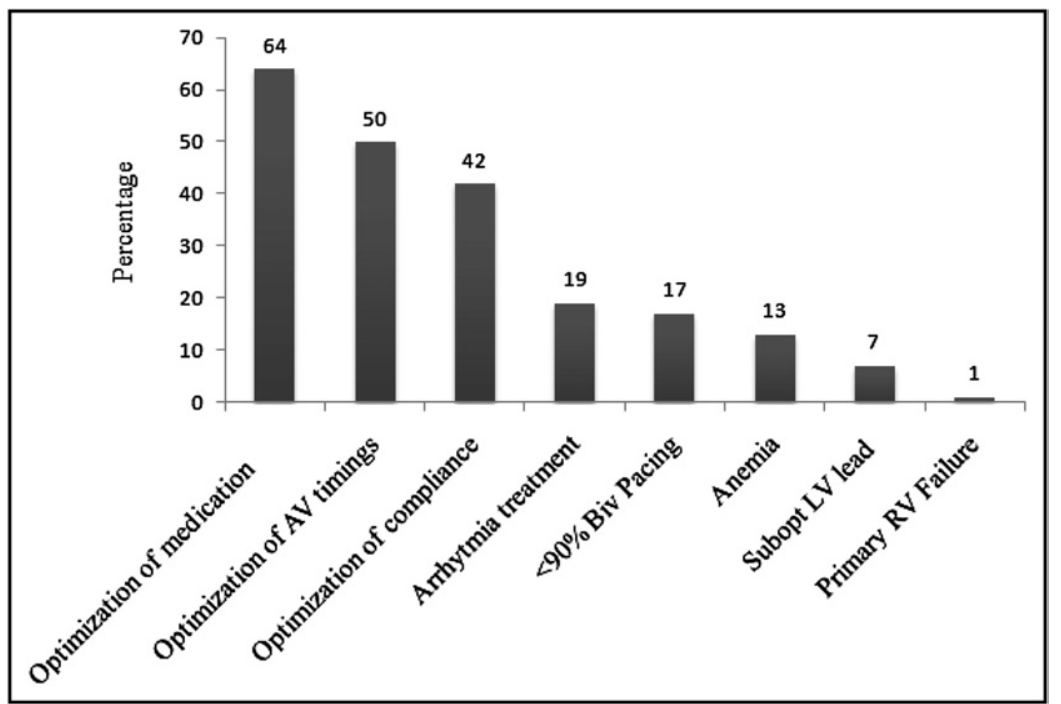

Figure 3. Interventions performed during visit to protocol-driven optimization cardiac resynchronization therapy clinic. $\mathrm{RV}=$ right ventricular; Subopt $=$ suboptimal. Other abbreviations as in Figure 2.

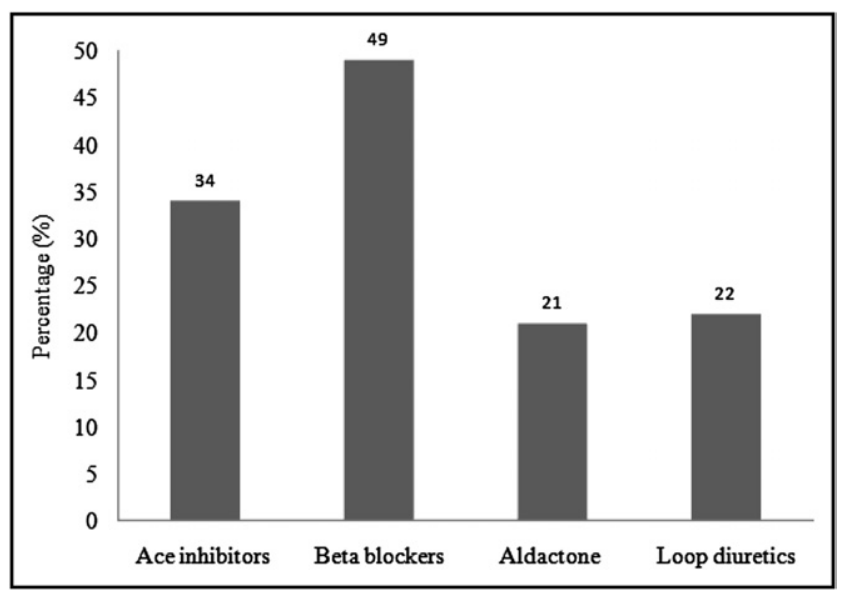

Figure 4. Bar graph presenting percentage of patients whose medication was optimized in the protocol-drive optimization cardiac resynchronization therapy clinic. Ace $=$ angiotensin-converting enzyme.

drugs was possible for $1 / 2$ the patient population, decrease in dosage of loop diuretics for $20 \%$ of patients, and $43 \%$ of patients confirmed to having poorly maintained their medication and dietary guidelines. In addition, $51 \%$ of patients were shown to be paced with suboptimal AV timings and $20 \%$ of patients presented with arrhythmias.

Positive remodeling was noted in the 2 groups with regard to $\mathrm{LV}$ dimension and $\mathrm{LV}$ function. However, extent of positive LV remodeling and improvement in LV ejection fraction were significantly greater in the group receiving a protocol-driven approach from the start (Figure 5). Moreover, as presented in Table 2, improvement in maximum exercise capacity as measured through maximum oxygen consumption was more pronounced for the protocol-driven group. Interestingly, these positive effects did not seem to relate to a greater decrease in dyssynchrony because the decrease in QRS and PR times did not differ between groups.

At the end of the follow-up period (mean follow-up duration $19 \pm 11$ months for the 2 groups), $36 \%$ of patients had

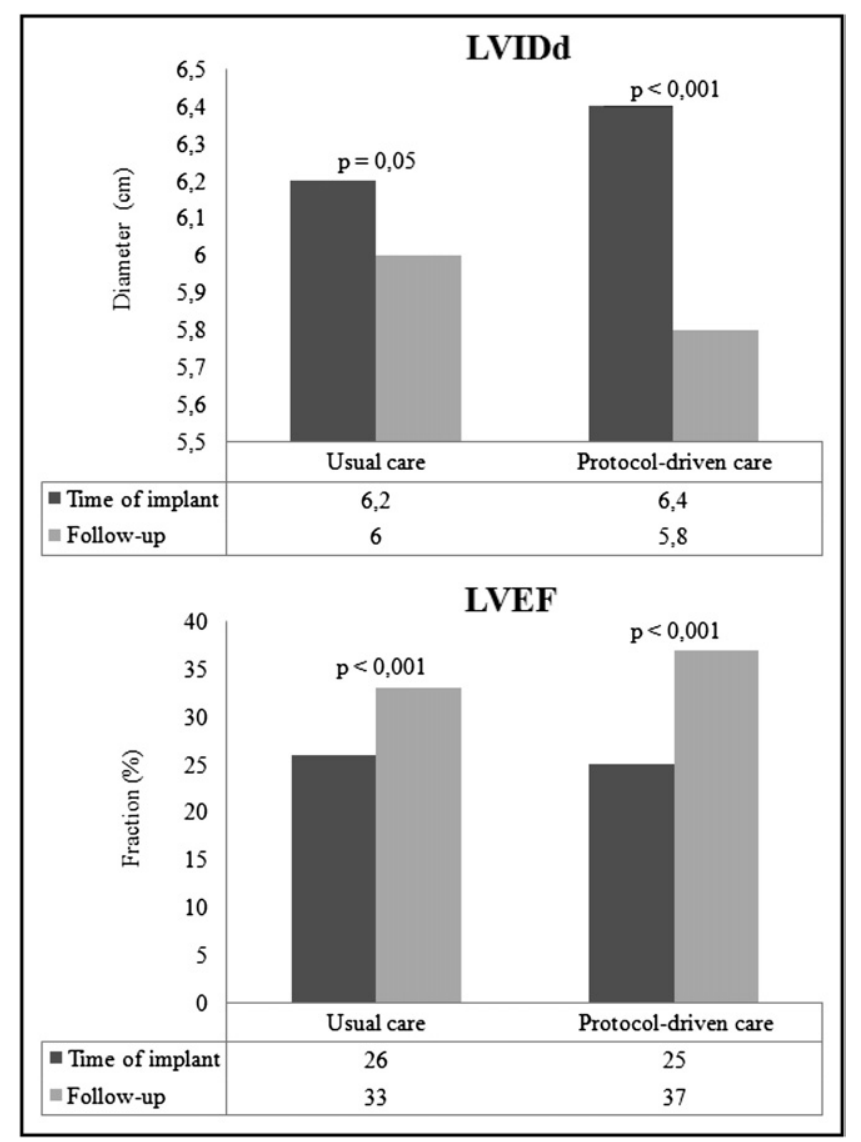

Figure 5. Bar graph for left ventricular internal diameter in diastole (centimeters) and left ventricular ejection fraction (percentage) at time of implantation versus follow-up for the 2 study groups. Abbreviations as in Figure 2.

died, undergone cardiac transplantation, and/or were hospitalized for decompensated heart failure. Although overall mortality/cardiac transplantation was similar for the 2 groups ( 3 vs 4 events, $\mathrm{p}=1$ ), patients receiving protocol-driven care had 
Table 2

Clinical data

\begin{tabular}{lccc}
\hline & $\begin{array}{c}\text { Time of } \\
\text { Implantation }\end{array}$ & Follow-Up & p Value \\
\hline $\begin{array}{l}\text { Echocardiographic data } \\
\text { Left ventricular internal diastolic } \\
\quad \text { diameter (cm) }\end{array}$ & & & \\
$\quad$ Usual care & $6.2 \pm 0.8$ & $6.0 \pm 1.0$ & 0.05 \\
$\quad$ Protocol-driven care & $6.4 \pm 1.0$ & $5.8 \pm 0.9$ & $<0.001$ \\
Left ventricular ejection fraction & & & \\
$\quad$ (\%)* & & & \\
$\quad$ Usual care & $26 \pm 8$ & $33 \pm 9$ & $<0.001$ \\
$\quad$ Protocol-driven care & $25 \pm 8$ & $37 \pm 9$ & $<0.001$ \\
Cycloergometric data & & & \\
Maximum volume (ml/kg/min)* & & & \\
$\quad$ Usual care & $14.1 \pm 3.2$ & $14.5 \pm 2.5$ & $\mathrm{NS}$ \\
$\quad$ Protocol-driven care & $14.0 \pm 4.8$ & $15.5 \pm 4.2$ & 0.03 \\
Maximum heart rate (beats/min) & & & \\
$\quad$ Usual care & $117 \pm 18$ & $104 \pm 22$ & $\mathrm{NS}$ \\
$\quad$ Protocol-driven care & $115 \pm 24$ & $105 \pm 26$ & 0.02 \\
Electrocardiographic data & & & \\
PR width (ms) & & & \\
$\quad$ Usual care & $187 \pm 45$ & $142 \pm 26$ & $<0.001$ \\
$\quad$ Protocol-driven care & $188 \pm 46$ & $128 \pm 30$ & $<0.001$ \\
QRS width (ms) & & & \\
$\quad$ Usual care & $155 \pm 32$ & $149 \pm 30$ & $\mathrm{NS}$ \\
Protocol-driven care & $157 \pm 29$ & $150 \pm 21$ & $\mathrm{NS}$ \\
\hline
\end{tabular}

* There was a significant difference between the 2 study groups at follow-up.

fewer adverse events during follow-up ( 28 vs 9 events, $p$ $<0.001$; Figure 6). The protocol-driven follow-up was not associated with lower all-cause mortality (odds ratio 1.085, $95 \%$ confidence interval 0.231 to $5.084, \mathrm{p}=\mathrm{NS}$ ) but did lead to a decrease in heart failure hospitalization (odds ratio 0.137 , $95 \%$ confidence interval 0.056 to $0.335, \mathrm{p}<0.001$ ) compared to routine care after implantation.

\section{Discussion}

The potential benefit of heart failure management in addition to a comprehensive protocol-driven CRT optimization strategy has been demonstrated in patients without robust clinical or echocardiographic responses to CRT. ${ }^{1} \mathrm{We}$ report for the first time a potential incremental benefit of such a CRT optimization strategy immediately after implantation of CRT, which is in part driven not only by device optimization but also by judicious uptitration of neurohormonal blockers and decreases in loop diuretics and heart failure education in close coordination of care with primary caregivers.

As in other interventions, extent of response to CRT can be heterogenous, and there is ongoing debate regarding the best strategies to optimize device performance. Much attention has focused primarily on refining preimplantation patient selection to predict a favorable response or on improving techniques and determination of optimal lead positioning to achieve maximal synchronization. However, maximizing benefits of CRT for those with implants is particularly important because CRT is an invasive intervention with known complications. Our group previously demonstrated in a systematic evaluation of implementation that nonresponding patients in a large subset of patients achieved substantial benefit by a combination of device adjustments and standard components of heart failure management interventions. ${ }^{1}$ We extend our findings to all patients immediately after CRT implantation in which immediate assessment of device parameters guided by clinical and echocardiographic evaluation may be associated with incremental clinical and echocardiographic responses that are directly associated with better long-term outcomes. Similar to our previous findings in the nonresponder population, a large majority of issues identified included presence of rhythm abnormalities (19\%) with concomitant inadequate delivery of biventricular pacing, improving compliance in decreasing salt and fluid intakes (42\%), optimization of medical management as an adjunct to device adjustments (64\%), and echocardiographically guided AV-optimization $(50 \%)$. These observations were unlikely biased by selection because the indication, implantation, and postprocedure care were provided by the same personnel with the exception of the additional upfront protocol-driven evaluation. Hence, our observations highlight the notion that current postimplantation approaches to longitudinal monitoring may overlook important issues such as optimization of medical therapy and heart failure education.

One of the most intriguing findings in our report is that for the first time we observed that up to $2 / 3$ of patients tolerated uptitration of neurohormonal blockers after CRT implantation to dosages previously not tolerated before CRT implantation. There may be several reasons that may explain this observation. First, the ability for CRT to provide hemodynamic augmentation in the setting of dyssynchrony has been well described in the literature and has been the basis for its indication. ${ }^{1,6,7}$ Second, the dependency of diuretic therapy to relieve congestion may be decreased; hence, adjustments of diuretic therapy may be necessary to better optimize the filling pressures needed to promote recovery. The 2 effects can commence immediately after CRT and perhaps more promptly after better adjustments of atrioventricular delay to establish the most optimal mitral inflow pattern. The ability to increase transition of care from time of discharge after device implantation to long-term management of heart failure likely further facilitates long-term improvement.

The design of our protocol-driven follow-up of ambulatory patients implanted with a CRT is unique yet scalable to broad adoption. First, it commenced immediately after implantation in all patients with implanted CRT, thus providing an upfront assessment and intervention that may benefit a larger proportion of patients especially when applied even at the time of discharge from hospitalization for implantation. Second, it used a combination of readily applicable (rather than research-based) testing and interventions that is familiar to patients and health care providers in a manner similar to our approach in the nonresponder population. ${ }^{1}$

Although positive effects toward improvement in exercise capacity and remodeling were noticeable in the 2 groups, the extent of these positive effects was significantly greater with the protocol-driven approach, ultimately leading to a decreased incidence of adverse events at follow-up. Interestingly, this was not attributable to a more pronounced decrease in electrical dyssynchrony in the protocol-driven 


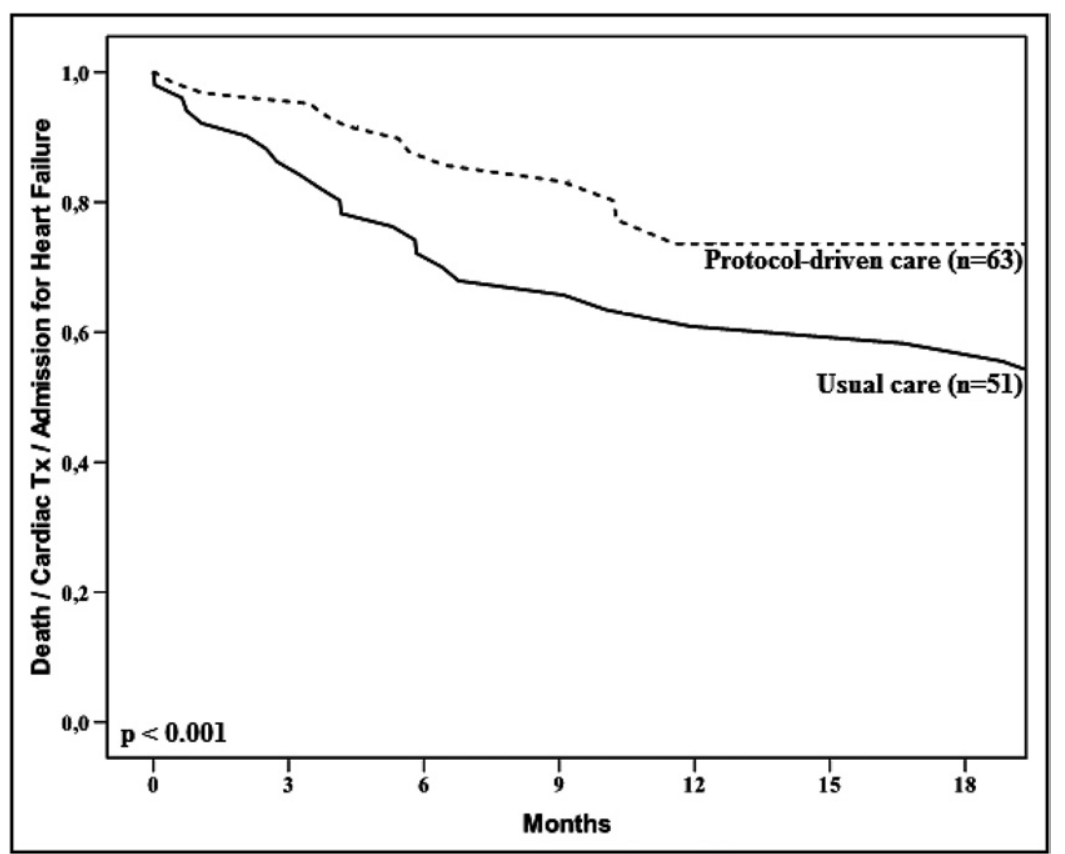

Figure 6. Kaplan-Meier survival curve for clinical outcomes of usual cardiac care versus protocol-driven care with combined end point of death, cardiac transplantation (Tx), and admission for heart failure.

approach. It is therefore conceivable that nondevice-related contributors of CRT response that can act independently of (but synergistically to) cardiac resynchronization will directly influence clinical and echocardiographic responses to CRT. Because contemporary postimplantation patient management is evolving more and more toward remote evaluation and monitoring, our data may argue conversely that an improvement in clinical or echocardiographic response after successful resynchronization should not imply that a routine follow-up visit or just a remote device follow-up is sufficient. Instead, it is conceivable that an upfront comprehensive heart failure management approach is warranted in patients receiving CRT to ensure a more persistent and meaningful alteration in the natural history of heart failure, thus maximizing chances to delay disease progression.

It is important to recognize that this is a single-center experience and not a randomized but a historical control comparison of 2 treatment strategies. Nevertheless, the period was selected because implantation indications and techniques were relatively mature and care patterns delivered by health care providers (including indications for medical and device therapies) were largely unchanged during this period.
1. Mullens W, Grimm RA, Verga T, Dresing T, Starling RC, Wilkoff BL, Tang WH. Insights from a cardiac resynchronization optimization clinic as part of a heart failure disease management program. J Am Coll Cardiol 2009;53:765-773.

2. Schiller NB, Shah PM, Crawford M, DeMaria A, Devereux R, Feigenbaum H, Gutgesell H, Reichek N, Sahn D, Schnittger I. Recommendations for quantitation of the left ventricle by two-dimensional echocardiography. American Society of Echocardiography Committee on Standards, Subcommittee on Quantitation of Two-Dimensional Echocardiograms. J Am Soc Echocardiogr 1989;2:358-367.

3. Mullens W, Tang WH, Grimm RA. Using echocardiography in cardiac resynchronization therapy. Am Heart J 2007;154:1011-1020.

4. Grimm R. Non-responders and patient selection from an echocardiographic perspective. In: Sutton J, ed. Cardiac Resynchronization Therapy. London, UK: Informa UK, 2007:251-261.

5. Gorcsan J, Abraham T, Agler DA, Bax JJ, Derumeaux G, Grimm RA, Martin R, Steinberg JS, Sutton MS, Yu CM. Echocardiography for cardiac resynchronization therapy: Recommendations for performance and reporting-a report from the American Society of Echocardiography Dyssynchrony Writing Group endorsed by the Heart Rhythm Society. J Am Soc Echocardiogr 2008;21:191-213.

6. Kass DA. Highlighting the R in CRT. Circulation 2007;116:14341436.

7. Mullens W, Verga T, Grimm R, Starling R, Wilkoff B, Tang W. Persistent hemodynamic benefits of cardiac resynchronization therapy with disease progression in advanced heart failure. J Am Coll Cardiol 2009;53:589-596. 